\title{
Room temperature magnetic imaging of magnetic storage media and garnet epilayers in the presence of external magnetic fields using a sub-micron GaAs SHPM
}

\author{
Adarsh Sandhu $^{\mathrm{a}, *}$, Hiroshi Masuda ${ }^{\mathrm{b}}$, Ahmet Oral ${ }^{\mathrm{c}}$, Simon.J. Bending ${ }^{\mathrm{d}}$ \\ ${ }^{a}$ Department of Electrical Engineering, Tokai University, 1117 Kitakaname, Hiratsuka, 259-1292, Japan \\ ${ }^{\mathrm{b}}$ Toei Kogyo Ltd., 8-13-1, Tadao, Machida, 194-0035, Japan \\ ${ }^{\mathrm{c}}$ Department of Physics, Bilkent University, 06533 Ankara, Turkey \\ ${ }^{\mathrm{d}}$ Department of Physics, University of Bath, Claverton Down, BA2 7AY, UK
}

\begin{abstract}
A multipurpose room temperature scanning Hall probe microscope (RT-SHPM) system incorporating an ultra-high sensitive sub-micron GaAs Hall probe (active area of $\sim 0.8 \times 0.8 \mu \mathrm{m}^{2}$; room temperature Hall coefficient of $0.3 \Omega / \mathrm{G}$ ) exhibiting extremely high magnetic field sensitivity $(0.04 \mathrm{G} / \sqrt{ } \mathrm{Hz})$ was used for the direct, non-invasive and quantitative imaging of magnetic field fluctuations in very close proximity to the surfaces of thin film magnetic storage media, Bi substituted iron garnet epilayers and demagnetized Sr-ferrite permanent magnets placed in external magnetic fields. A scanning tunnelling microscope tip integrated adjacent to the Hall probe was used for precise vertical positioning of the probe. RT-SHPM images of 1.4 MB written floppy disks clearly showed well-defined magnetic transitions to coalesce into small island-like structures under external perpendicular fields greater than 1300 Oe. The RT-SHPM is demonstrated to be a versatile means of quantitatively monitoring micron-sized magnetic domain structures in the presence of external magnetic fields at room temperature. (C) 2001 Elsevier Science B.V. All rights reserved.
\end{abstract}

PACS: $75.70 . \mathrm{Kw} ; 75.60 . \mathrm{Ch} ; 75.50 . \mathrm{Ss} ; 85.70 . \mathrm{Ge} ; 85.30$

Keywords: A1. Scanning hall probe microscope; A1. Magnetic fields; A3. Molecular beam epitaxy; B2. Magnetic materials; B3. Hall effect sensor; B3. Heterojunction semiconductor devices

\section{Introduction}

The development of magnetic information storage technology, optical isolation devices and high coercivity permanent magnets requires a fundamental knowledge of the physical properties

\footnotetext{
*Corresponding author. Fax: + 81-463-58-9203.

E-mail address: sandhu@keyaki.cc.u-tokai.ac.jp (A. Sandhu).
}

of magnetic domains. Historically, the experimental observation of magnetic domain structures has been an extremely active area of research with early reports of the visualization of domains in single crystal silicon iron and their correlation with magnetization reported by Williams and Shockley in 1949 [1].

Modern-day methods employed for monitoring magnetic domains include semiconductor Hall bar sensors [2-8], magnetic force microscopy (MFM) 
[9], SQUID technology [10], Kerr and Faraday effect microscopy [11] and Bitter patterns using $\mathrm{Fe}_{3} \mathrm{O}_{4}$ particles suspended in colloids [12]. More enterprising methods include the use of anaerobic magnetotactic bacteria for domain visualization [13]. The aforementioned techniques have certain limitations with a restricted range of industrial applications. Some of the preferred characteristics of a more practical magnetic imaging method include: (a) non-invasive, quantitative imaging in the presence of external magnetic fields; (b) high sensitivity; (c) operation at room temperature.

In this paper, we describe the wide range of possible applications of a multipurpose room temperature scanning Hall probe microscope system (RT-SHPM) exhibiting the aforementioned characteristics. Samples studied included commercially available floppy disk media, single crystal garnets and demagnetized Sr-ferrite magnets.

\section{Experimental procedure}

\subsection{Components of RT-SHPM system}

The magnetic imaging system described in this study was specifically designed for operation at room temperature for scans up to $50 \times 50 \mu \mathrm{m}^{2}$. A GaAs/AlGaAs heterostructure micro-Hall probe sensor (HP) was mounted onto a piezoelectric scanning tube (PZT) at a tilt angle of $1.5^{\circ}$ with respect to the sample. A scanning tunnelling microscope (STM) tip integrated adjacent to the HP was used for precise vertical positioning. Magnetic imaging was carried out by first using a combination of high resolution stepper motors (coarse) and the PZT (fine) to position the HP into close proximity to the sample surface until a tunnel current was detected at the STM tip. Next, the HP was scanned over the surface while simultaneously measuring changes in Hall voltage that are proportional to fluctuations of the perpendicular component of the magnetic field emanating from the surface. A combination of the dimensions of the active area of the HP and distance from the sample surface determine the spatial resolution of the scan.
The design of the RT-SHPM system was based on a low temperature version previously reported [7] but with the following major modifications and additional features: (i) the tilt angle between the Hall probe and sample surface was precisely adjusted using an electronic angle sensor coupled to the PC control software; (ii) coarse sample approach was achieved by a high resolution motorized $\mathrm{z}$-stage with a $25 \mathrm{~mm}$ range; (iii) scan range up to $50 \times 50 \mu \mathrm{m}^{2}$; (iv) the use of electrodes on the reverse-side of the Hall probe chip carrier for electrical connection of the Hall bar and STM tip to the control and biasing electronics; (v) data acquisition with a choice of 3 modes including the STM/SHPM mode where scanning is carried out while simultaneously monitoring STM tip tunnel current and the real time mode where a $128 \times 128$ pixel scan is possible in about $1 \mathrm{~s}$; (vi) a completely new electronic control system incorporating an ultra stable DC current source and low noise amplifier circuits for improved $\mathrm{S} / \mathrm{N}$ of the measured Hall voltage at room temperature.

\subsection{MBE growth and fabrication of micro-hall probes}

A wide range of MBE growth conditions and heterostructure designs were investigated. The spatial resolution of SHPM magnetic images depends on the size of the active area of the Hall probe and the distance of the conducting channel, i.e., the $2 \mathrm{DEG}$, from the sample surface. It was found that the structure shown in Fig. 1 produced the best imaging results where the $2 \mathrm{DEG}$ electron density and mobility at $4.2 \mathrm{~K}$ were $2 \times 10^{11} \mathrm{~cm}^{-2}$ and $400,000 \mathrm{~cm}^{2} / \mathrm{Vs}$, respectively. The $2 \mathrm{DEG}$ is located approximately $100 \mathrm{~nm}$ below the surface of the epilayer. Shallower 2DEG locations led to degradation of electron mobility. For comparison, a set of Hall probe arrays were fabricated using GaAs/AlGaAs heterostructures grown by MOCVD but without satisfactory results. Our experiments thus far, suggest that MBE wafers are the preferred choice for the fabrication of high performance micro-Hall probes.

The Hall probe was fabricated by photolithography and was located $13 \mu \mathrm{m}$ away from the corner of an etched deep mesa that was coated 


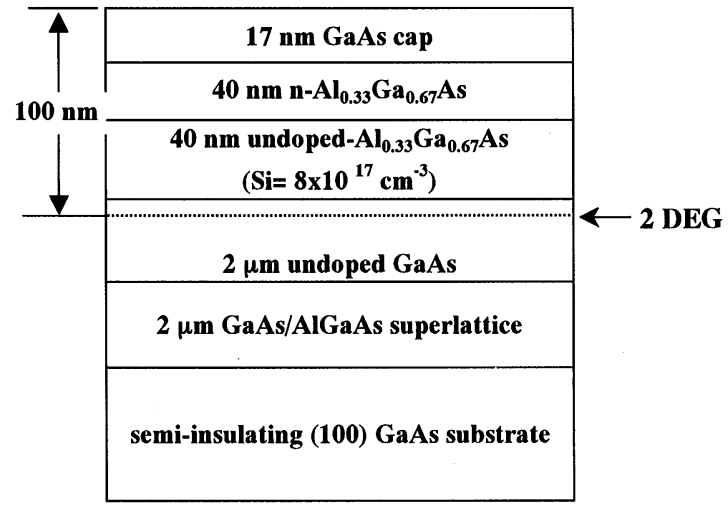

Fig. 1. MBE grown GaAs/AlGaAs heterostructure used for fabricating micro Hall probe.

with a thin gold layer to act as the STM tip. The STM tip was not coupled to the Hall bar thus reducing noise during measurement. The Hall probe had an active area of $\sim 0.8 \times 0.8 \mu \mathrm{m}^{2}$. The room temperature Hall coefficient of the HP was $0.3 \Omega / \mathrm{G}$ and the field sensitivity was $0.04 \mathrm{G} / \sqrt{ } \mathrm{Hz}$. A Hall driving current of $3 \mu \mathrm{A}$ was used for all the measurements described.

\subsection{Data visualization}

The raw two-dimensional image data of RTSHPM scans was stored as either a bit-map or ASCII format. In some cases, a three dimensional visualization of the SHPM image can be more informative for interpreting results. For such purposes, we developed unique program routines for the static and animated 3D visualization of SHPM data using Interactive Data Language (IDL v.5.3) [14].

\section{Results and discussion}

\subsection{Longitudinal recording media}

Digital magnetic recording systems employ recording heads to write (switch the direction of the magnetization by rapid pulsed changes in the direction of current in the recording head) magnetic regions in high coercivity thin film/ particulate recording media (cobalt alloys) moving relative to the write head. The magnetization of the magnetic regions resulting from the action of the write head is parallel to the film surface with stray surface flux emanating from magnetically charged transitions in magnetization ${ }^{1}$. The spatial and intensity distribution of the perpendicular components of the stray surface flux are sensed and imaged by the micro-Hall probe of the RTSHPM.

\subsection{1. 'Zip' disk media}

Fig. 2 shows a typical $50 \mu \mathrm{m} \times 50 \mu \mathrm{m}$ RT-SHPM image of a commercial $100 \mathrm{MB}$ 'Zip' disk and corresponding field variation along one of the tracks. The measurement was carried out in the STM/SHPM mode. The black and white regions correspond to stray surface fields alternating in sign and the transitions are seen to be nonuniform with magnetic fields ranging between $\pm 58 \mathrm{G}$.

\subsection{MB floppy disks}

Fig. 3 is a $25 \mu \mathrm{m} \times 25 \mu \mathrm{m}$ images of a track written on a commercially available $1.4 \mathrm{MB}$ floppy disk obtained using the STM/SHPM mode. Written transitions spaced approximately $2 \mu \mathrm{m}$ apart can be clearly observed and our results revealed them to vary between $\pm 130 \mathrm{G}$ from black to white. The 'noisy' area to the right-hand side of the transitions is the unwritten region between tracks.

Fig. 4(a)-(g) are seven representative $25 \mu \mathrm{m} \times 25 \mu \mathrm{m}$ images of the written floppy disk placed in an increasing external magnetic field applied perpendicular to the disk surface. The HP was positioned $0.8 \mu \mathrm{m}$ above the disk and the surface imaged continuously as the external field was increased from zero to a maximum of 3460 Oe in steps of 150 Oe. Each image frame was acquired in approximately $20 \mathrm{~s}$. The initial distinct magnetic transitions are seen to deform and coalesce into small islands under external fields greater than 1300 Oe. Fig. 5 shows the corresponding changes in the local fields along the lines superimposed on

\footnotetext{
${ }^{1}$ Research Systems, Inc., 4990 Pearl East Circle, Boulder, USA.
} 

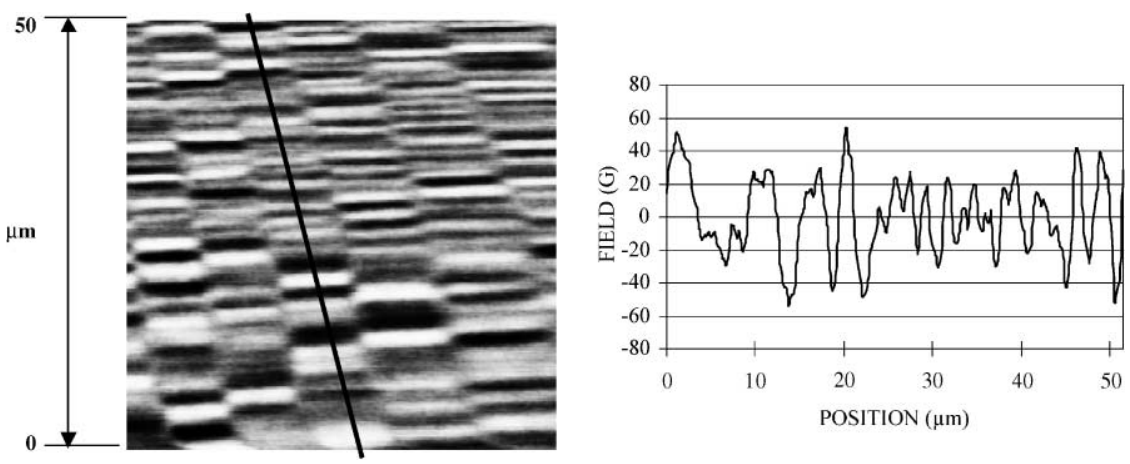

Fig. 2. $50 \mu \mathrm{m} \times 50 \mu \mathrm{m}$ RT-SHPM image of a $100 \mathrm{MB}$ 'zip' disk and field variation along a track.
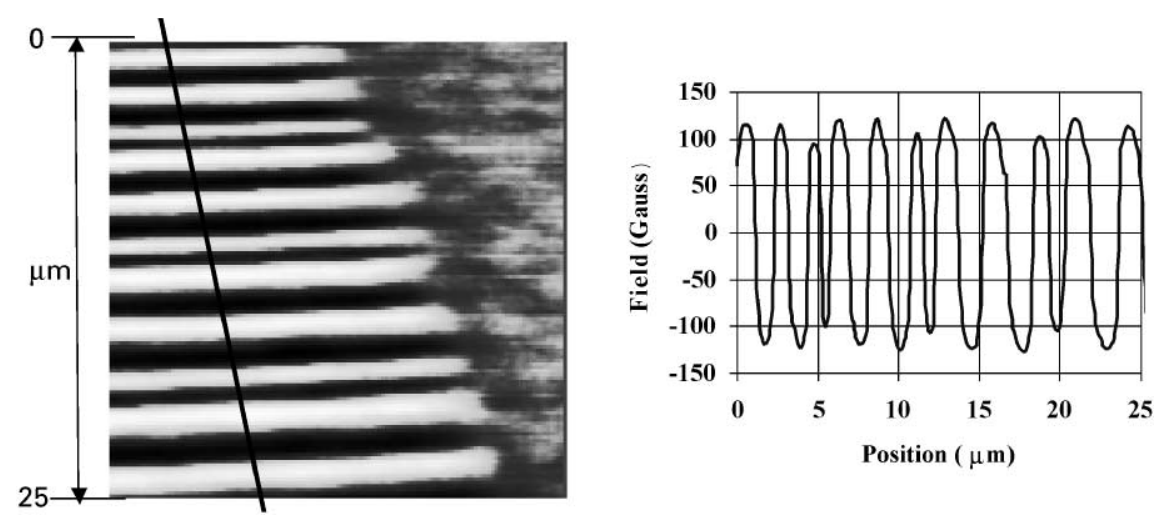

Fig. 3. $50 \mu \mathrm{m} \times 50 \mu \mathrm{m}$ RT-SHPM image of a written track on a $1.4 \mathrm{MB}$ floppy disk measured in SHPM/STM mode.

the images. The field measured at point ' $A$ ' in Fig. 4(a) decreases from $60 \mathrm{G}$ to less than $10 \mathrm{G}$ as the external applied field is increased from 593 to $3460 \mathrm{Oe}$. We are currently carrying out a detailed study using a vibrating sample magnetometer (VSM) and micro magnetic simulations in order to physically explain these RT-SHPM observations.

\subsection{Bismuth substituted iron garnet epilayers}

Fig. 6 shows $25 \mu \mathrm{m} \times 25 \mu \mathrm{m}$ images of a $5.5 \mu \mathrm{m}$ thick bismuth substituted iron garnet epilayer placed in a cyclic external perpendicular field as indicated. The images clearly show the initial hexagonal bubble lattice to expand and transform into a maze pattern. The measured fields range between $\pm 59 \mathrm{G}$. These results show the configurational hysteresis of domain structures in lowcoercivity films with strong perpendicular anisotropy [15].

Fig. 7 shows a $50 \mu \mathrm{m} \times 50 \mu \mathrm{m}$ image of a $360 \mu \mathrm{m}$ thick $\mathrm{Bi}$ substituted iron garnet epilayer. The image resembles 'fujitsubo' (barnacles). The RTSHPM image reveals a very complicated spatial field distribution that cannot be observed using conventional optical techniques often used for characterization of garnets.

These results showing the effect of an external field on striped domains and eventual bubble collapse can be used for characterizing thin films with large perpendicular anisotropy and 


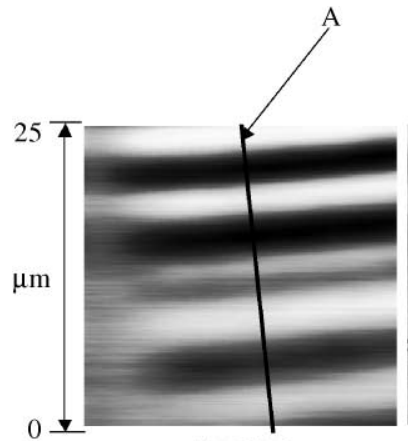

(a) $592 \mathrm{Oe}$

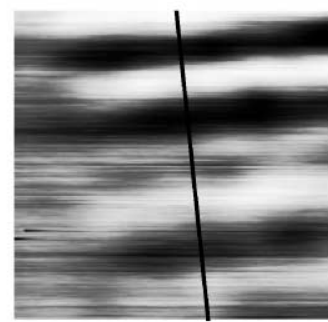

(e) $2480 \mathrm{Oe}$

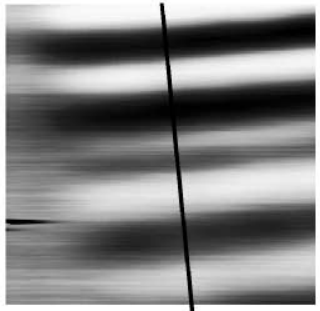

(b) $1247 \mathrm{Oe}$

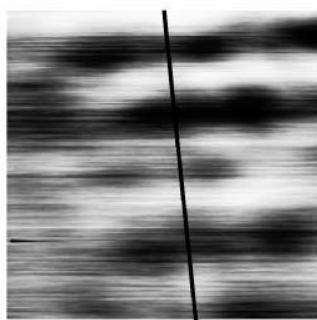

(f) $2960 \mathrm{Oe}$

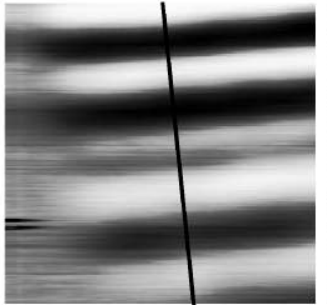

(c) $1519 \mathrm{Oe}$

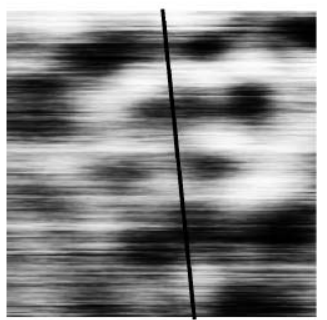

(g) $3460 \mathrm{Oe}$

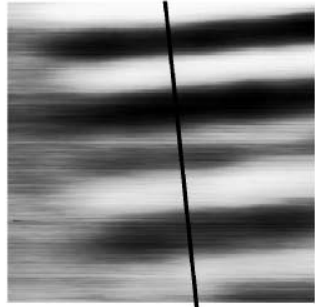

(d) $2050 \mathrm{Oe}$

Fig. 4. (a)-(g) $25 \mu \mathrm{m} \times 25 \mu \mathrm{m}$ images of written $1.4 \mathrm{MB}$ floppy disk in an external magnetic field. The numerals under the respective images indicate the applied external fields.

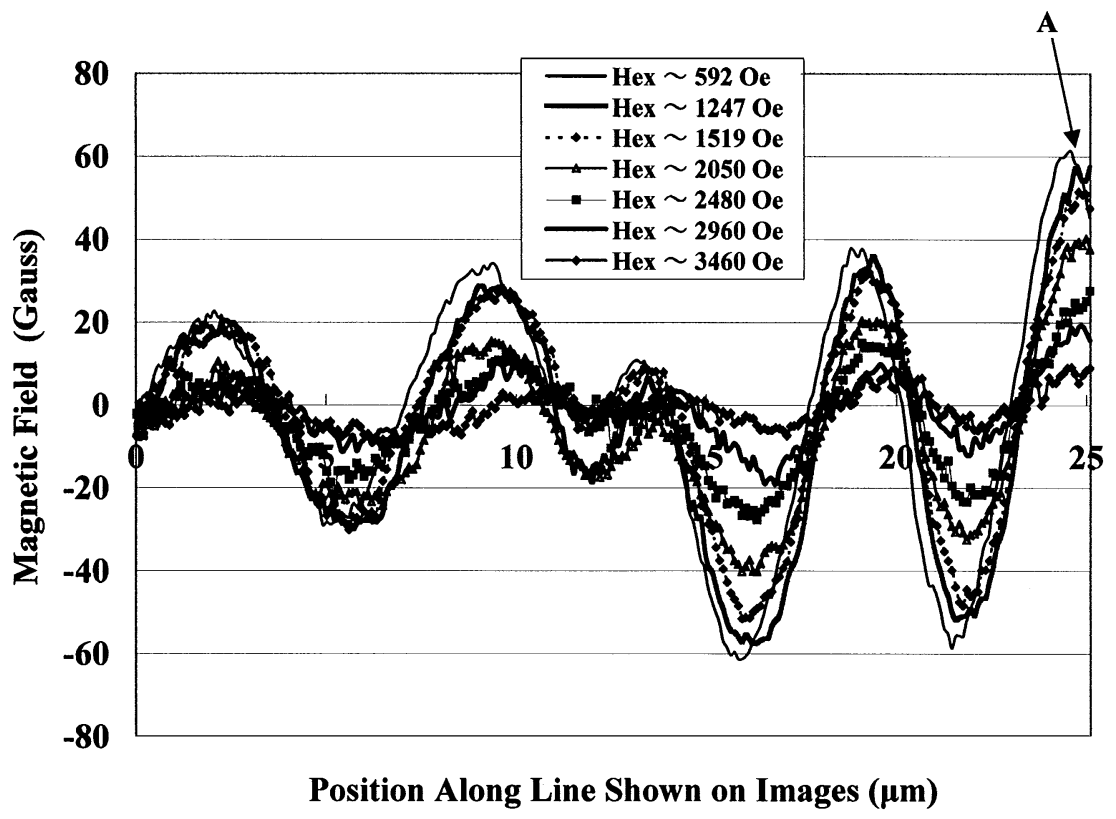

Fig. 5. Cross sectional variation of magnetic field along lines shown in in Fig. 3(a)-(g). 


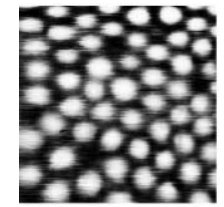

290 Oe

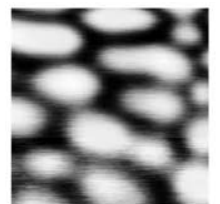

850 Oe

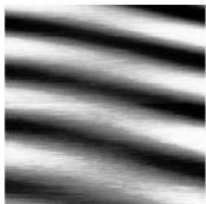

933 Oe

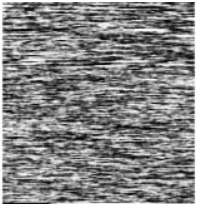

1131 Oe

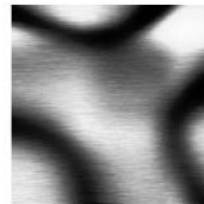

1027 Oe

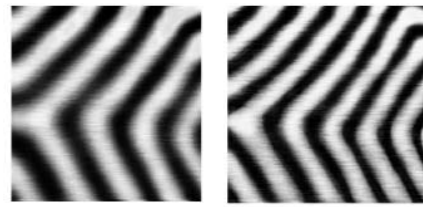

747 Oe

$160 \mathrm{Oe}$

Fig. 6. $25 \mu \mathrm{m} \times 25 \mu \mathrm{m}$ images of a $5.5 \mu \mathrm{m}$ thick single crystal bismuth substituted iron garnet epilayer placed in a cyclic external perpendicular field.

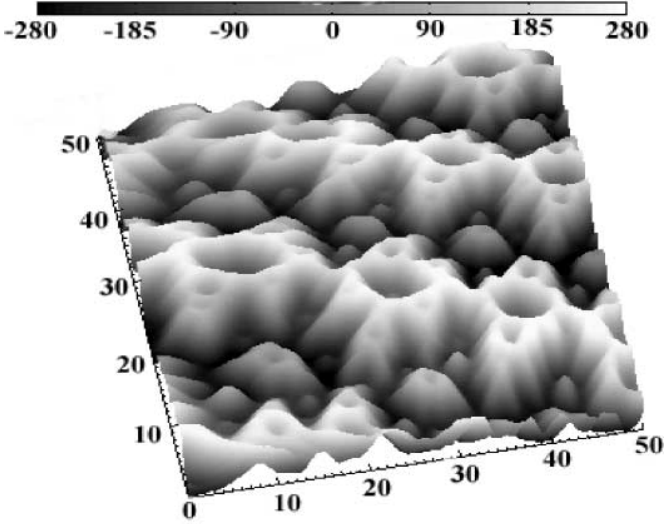

Fig. 7. $50 \mu \mathrm{m} \times 50 \mu \mathrm{m}$ 'fujitsubo-like' images of surface field fluctuations of a $360 \mu \mathrm{m}$ thick $\mathrm{Bi}$ substituted iron garnet epilayer.

determining parameters such as wall energy and saturation magnetization without the need for magnetometer measurements [16].

\subsection{Strontium ferrite permanent magnets}

Fig. 8(a) and (b) show the RT-SHPM images of a demagnetized $\mathrm{Sr}$ ferrite magnet without and with $(1760 \mathrm{Oe})$ an external perpendicular field, respectively. Domain structures are clearly imaged and are seen to increase in size on application of the external field. We are carrying out further RTSHPM analysis of domain wall movement in ferrite and other permanent magnets for a deeper understanding of the relationship between domain size and coercivity and saturation magnetization for the development of high performance permanent magnets [17].
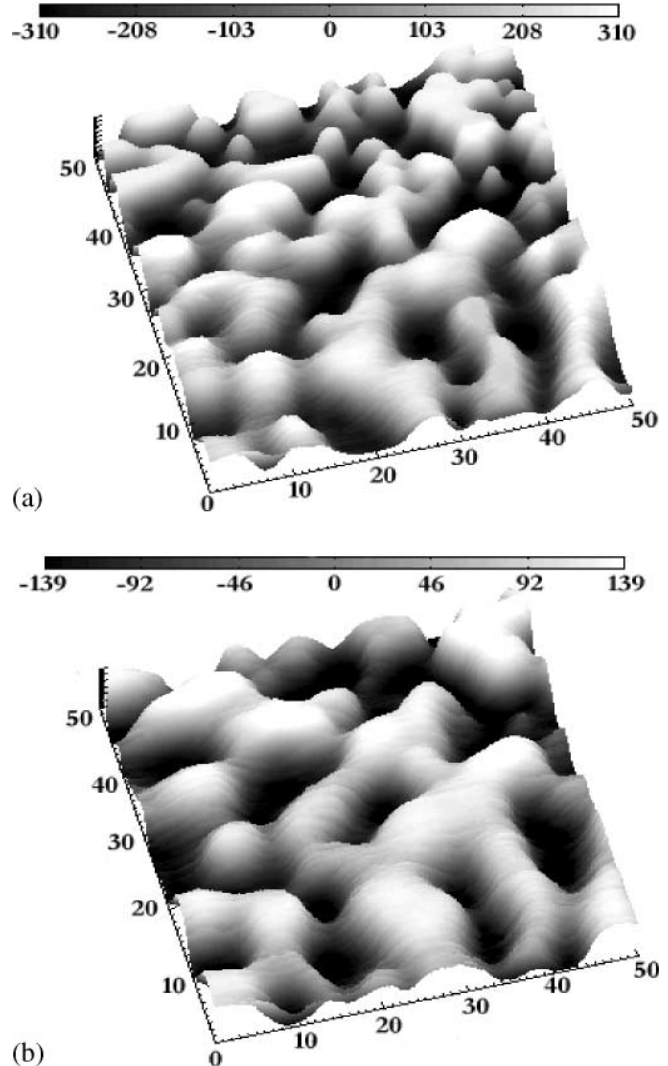

Fig. 8. (a) RT-SHPM image of a demagnetized $\mathrm{Sr}$ ferrite magnet without an external field applied. (b) RT-SHPM image of a demagnetized $\mathrm{Sr}$ ferrite magnet with an applied field of 1760 Oe.

\section{Summary}

A new versatile room temperature scanning Hall probe microscope system was used for the quantitative magnetic imaging of storage media, 
garnets and demagnetized strontium ferrite magnets. The RT-SHPM system is expected to find a wide range of applications in the development of information storage media and permanent magnets as well as new technology related to 'spintronics', where semiconductor/ferromagnetic hybrid structures are being studied for the next generation of magneto-electronic devices [18].

\section{References}

[1] H.J. Williams, W. Shockley, Phys. Rev. 75 (1949) 178.

[2] H.T. Coffey, Cryogenics 7 (1965) 73.

[3] M. Tinkham, J. Low Temp. Phys. (1971) 5465.

[4] M.A. Itzler, J.A. Simmons, P.M. Chaikin, Bull. Am. Phys. Soc. 36 (1991) 724.

[5] A.M. Chang, H.D. Hallen, L. Harriott, H.F. Hess, H.L. Kao, J. Kwo, R.E. Miller, R. Wolfe, J. van der Ziel, T.Y. Chang, Appl. Phys. Lett. 61 (1992) 1974.

[6] J. Siegel, J. Witt, N. Ventura, S. Field, Rev. Sci. Instrum. 66 (1995) 2520.
[7] A. Oral, S.J. Bending, M. Henini, J. Vac. Sci. Technol. B 14 (1996) 1202.

[8] T. Fukuhara, H. Sugawara, T. Hasegawa, K. Tanaka, H. Sakaki, T. Kimura, Y. Tokumura, Science 284 (1999) 1969.

[9] U. Hartmann et al., J. Magn. Magn. Mater. 101 (1991) 263.

[10] C.C. Tsuei, J.R. Kirtley, C.C. Chi, L.S. Yu-Jahnes, A. Gupta, T. Shaw, J.Z. Sun, M.B. Ketchen, Phys. Rev. Lett. 73 (1994) 593.

[11] J.M. Florczak, E.D. Dahlberg, J. Appl. Phys. 67 (1990) 7520 .

[12] G.C. Rauch, R.F. Krause, C.P. Izzo, K. Foster, W.O. Barlett, J. Appl. Phys. 55 (1984) 2145.

[13] G. Harasko, H. Pfutzner, K.. Futschik, Magn. Magn. Mater. 133 (1994) 409.

[14] S.X. Wang, A.M. Taratorin, Magnetic Information Storage Technology, Academic Press, New York, 1999.

[15] K.L. Babcock, R.M. Westervelt, Phys. Rev. A 40 (4) (1989) 2022.

[16] T.G.W. Blake, C.C. Shur, Y. Tu, E. Della Torre, IEEE Trans. Magn. 18 (1982) 985.

[17] H.R. Kirchmayr, J. Phys. D 29 (1996) 2763.

[18] P. Ball, Nature, 404 (2000) 918. 